\title{
Desorption Electrospray Ionization: Proteomics Studies by a Method That Bridges ESI and MALDI
}

\author{
Zoltan Takats, Justin M. Wiseman, Demian R. Ifa, and R. Graham Cooks
}

Adapted from "Desorption Electrospray lonization: Proteomics Studies by a Method That Bridges ESI and MALDI," Chapter 6, in Proteomics: Methods Express (eds. O'Connor and Hames). Scion Publishing Ltd., Oxfordshire, United Kingdom, 2007.

\section{INTRODUCTION}

Desorption electrospray ionization (DESI) is a desorption ionization (DI) method by nature, and, like matrix-assisted laser desorption/ionization (MALDI), it is used for the analysis of material present on a surface. DESI includes features reminiscent of electrospray ionization (ESI) in respect to both its instrumental and mechanistic aspects. However, the analyte in the DESI experiment is not in solution as in ESI. Instead, a microelectrospray ion source is used to produce charged droplets, ionic clusters, and/or gas-phase ions (depending on chosen experimental conditions), and these are directed at the sample surface. The sample is present in the ambient environment. An electrical potential of several kilovolts $(\mathrm{kV})$ is applied to the spray solution, and pneumatic nebulization is used to assist in desolvation. Ionization of molecules present on the sample surface occurs upon the impact of the ESI-originated, charged particles with the surface. Surfaces include deposited samples on sample holder targets as well as surfaces of natural objects such as biological tissues or minerals.

\section{RELATED INFORMATION}

The following DESI protocols are available in this issue of CSH Protocols: Desorption Electrospray Ionization (DESI) Analysis of Intact Proteins/Oligopeptides, Desorption Electrospray Ionization (DESI) Analysis of Tryptic Digests/Peptides, and In Situ Desorption Electrospray Ionization (DESI) Analysis of Tissue Sections.

\section{DESI INSTRUMENTATION}

A DESI ion source is a pneumatically assisted microelectrospray source equipped with a surface holder and positioning devices. The source comprises two main parts, a sprayer assembly and a surface assembly, both mounted on a source base (Fig. 1). High voltage (3-5 kV) is applied to the liquid junction on a stainless-steel union or on the stainless-steel syringe needle used to deliver the spray solvent. (Caution: The high-voltage electrical connection should be isolated from the environment to prevent electrical shock.) The sprayer itself is mounted onto a vertical rotating stage, which, in turn, is mounted onto a three-dimensional (3D) linear moving stage. The linear movement is used to change the sprayer-to-mass spectrometer or sprayer-to-sample distance, and also to compensate for the different angles at which the sprayer is used. The rotating stage allows selection of charged droplet impact angles of $0^{\circ}$ to $90^{\circ}$. The sample is placed on the surface holder, which is mounted onto a separate 3D moving stage. In one version, the surface holder can carry $5 \mathrm{~cm} \times 1 \mathrm{~cm}$ large disposable surface slides, which lie in a thin piece of stainless steel embedded into a surface holder made of polytetrafluoroethylene (PTFE). The metal support is connected to an external high-voltage power supply to provide an appropriate surface potential. The range of surface potentials available is identi- 


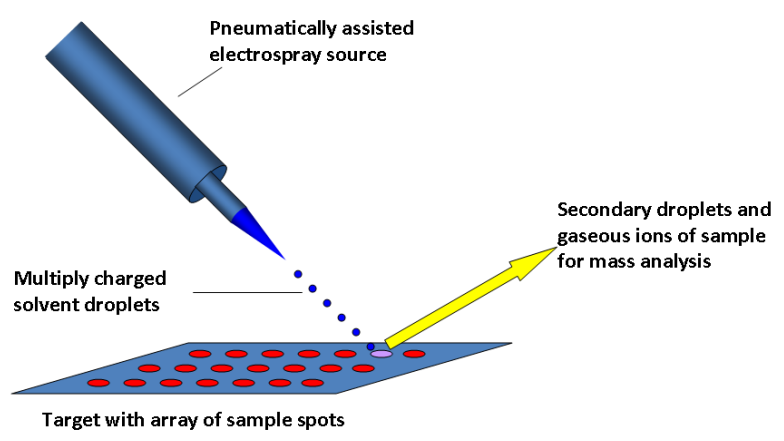

FIGURE 1. Concept of a DESI ion source. The DESI ion source is a pneumatically assisted microelectrospray ion source capable of delivering a coaxial sheath gas at up to $350 \mathrm{~m} / \mathrm{sec}$. High-voltage contact is applied at the liquid junction between solvent delivery lines or at the spray nozzle. The sample is in the ordinary ambient environment. (Reprinted with permission, (C) 2007 Scion Publishing Ltd.)

cal to the range for the ion source (0-6 kV). In a different surface holder design, the surface holder is a polyetheretherketone-coated aluminum block, which has a built-in heater cartridge, a Peltier cooling device, and a thermometer. This surface holder provides controlled surface temperatures in the range of $-20^{\circ} \mathrm{C}$ to $300^{\circ} \mathrm{C}$. The newest DESI ion sources (Omni Spray lon Source) feature two chargecoupled device cameras and a light source. For high-throughput applications, the surface assembly is replaced by a moving belt system or rotating disk system, as shown in Figure 2. Sample targets using pre-deposited arrays of samples similar to those used in commercial MALDI systems are also feasible, but the moving belt and rotating disk devices are mostly used in DESI.

A recent development in DESI allows for the direct analysis of microtiter well plates (e.g., $N=96$, 384 , etc.) in a high-throughput format. This advance was brought about by the addition of a small, pressure-tight enclosure surrounding the sprayer and inlet into the mass spectrometer (Fig. 3). This allows for effective sampling of ions even when the spray is orthogonal to the sample surface and with an inlet capillary parallel to the sprayer. An embodiment of this configuration allows for direct analysis inside well plates, where the well wall naturally creates the enclosure. This technique, referred to as geometry-independent DESI, promises to simplify DESI instrumentation and make it much easier to implement.

DESI ion sources can be coupled to practically any type of mass analyzer fitted with an atmospheric interface of the type used in electrospray or atmospheric pressure ionization sources. Most examples of spectra provided in this article and its related protocols (see Related Information) were recorded using a linear ion trap mass spectrometer (LTQ; Thermo Scientific), although in some cases, the data were recorded using a time-of-flight mass spectrometer.

\section{ION FORMATION}

The relationship of DESI to the other DI methods is close, at least at the phenomenological level. All of the DI methods (LDI, MALDI, plasma desorption [PD], secondary ion mass spectrometry [SIMS], and fast atom bombardment [FAB]) involve condensed-phase samples being impacted by projectiles; these include photons (laser desorption, including MALDI) and translationally excited atoms (FAB) and energetic ions (SIMS). The projectiles used in SIMS include polyatomic ions, and it is well established

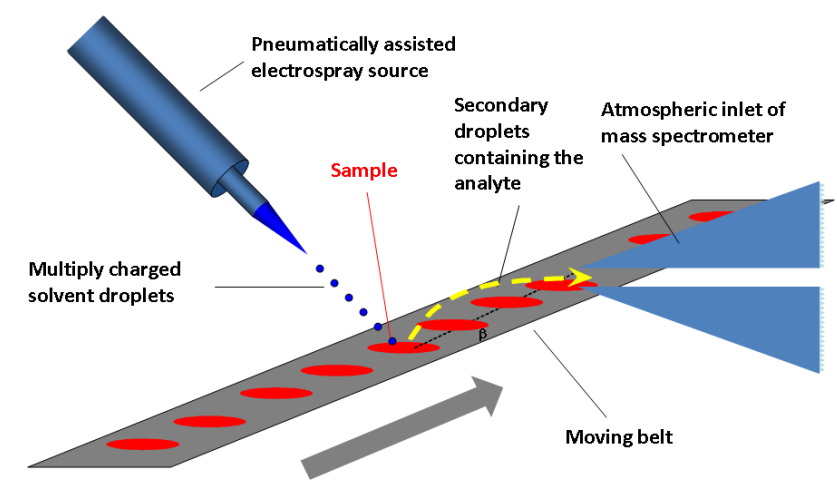

FIGURE 2. High-throughput DESI experiment. Samples are spotted on a moving belt positioned in front of the inlet of the mass spectrometer. (Reprinted with permission, (c) 2007 Scion Publishing Ltd.) 


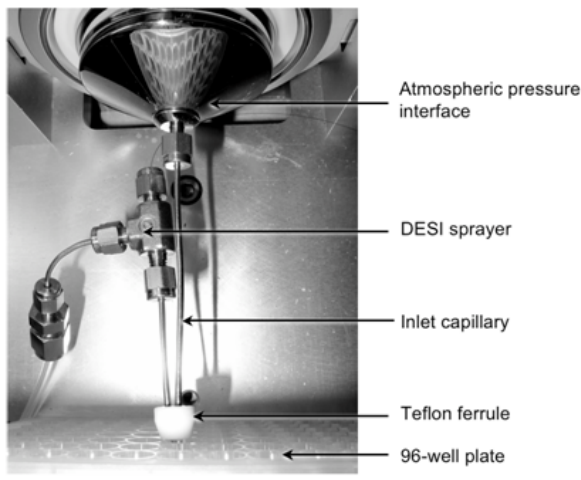

FIGURE 3. Picture of geometry-independent DESI ion source on a Thermo Scientific LTQ mass spectrometer. (Reprinted with permission, (C) 2007 Scion Publishing Ltd.)

that polyatomics are significantly more efficient than are atomic ions in sputtering molecules from surfaces. However, hitherto all DI experiments using particles as projectiles have been conducted in a high vacuum environment: The fact that DESI is performed in air is the principal feature that distinguishes it from earlier DI methods. This also implies significant differences in the fundamental processes involved, because the momentum transfer collisions upon which traditional SIMS and other DI sputtering mechanisms are based are not applicable in the high-pressure environment of DESI, since the projectiles can have only low kinetic energies. Low-energetic collisions (1-100 eV in the laboratory frame of reference), however, can effect desorption/ionization of molecules from surfaces through a heterolytic process known as chemical sputtering (Vincenti and Cooks 1988). In this process, desorption/ionization occurs as a result of charge transfer (electron, proton, or other ionic fragment) from a low translational energy primary ion to a surface molecule.

The collisions of multiply charged aqueous droplets with a surface introduce an additional and fundamentally different mechanism of ion formation in DESI. The multiply charged primary droplets impacting the surface are scattered as secondary droplets that carry the analyte and a fraction of the charge and mass of the primary droplet. In this case, the "desorption" event is essentially a phase transfer; the solid-phase analyte is adsorbed onto the impacting aqueous droplets and carried in solution to the mass spectrometer. In almost all cases, this is the primary desorption/ionization mechanism present in the DESI experiment (Venter et al. 2006). Subsequent ion formation from the secondary droplet closely resembles conventional ESI and proceeds either by ion emission (ion evaporation model) or by evaporation of neutral droplet molecules (charged residue model) to produce gaseous ions (see Fig. 4). Hence, DESI spectral characteristics are rather similar to those of ESI. DESI spectra of peptides and proteins feature a series of multiply charged molecular ions and almost completely lack ionization-related fragmentation (see Fig. 5).

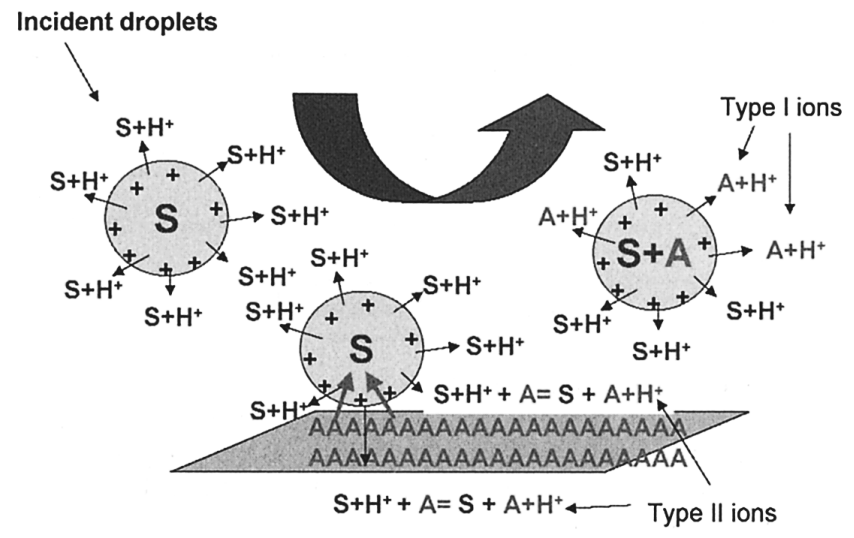

FIGURE 4. General scheme for two ion formation mechanisms in DESI-MS. Type I ions are formed by charge (e.g., proton) transfer when primary ions or multiply charged incident droplets impact the surface and react with the analyte present at the surface. Type II ions are formed when charged droplets impact the surface, dissolve the analyte, and leave the surface as secondary charged microdroplets, which are desolvated in the mass spectrometer inlet to give gas phase ions. (S) Surface; (A) analyte. (Reprinted with permission, (c) 2007 Scion Publishing Ltd.) 


\section{A}
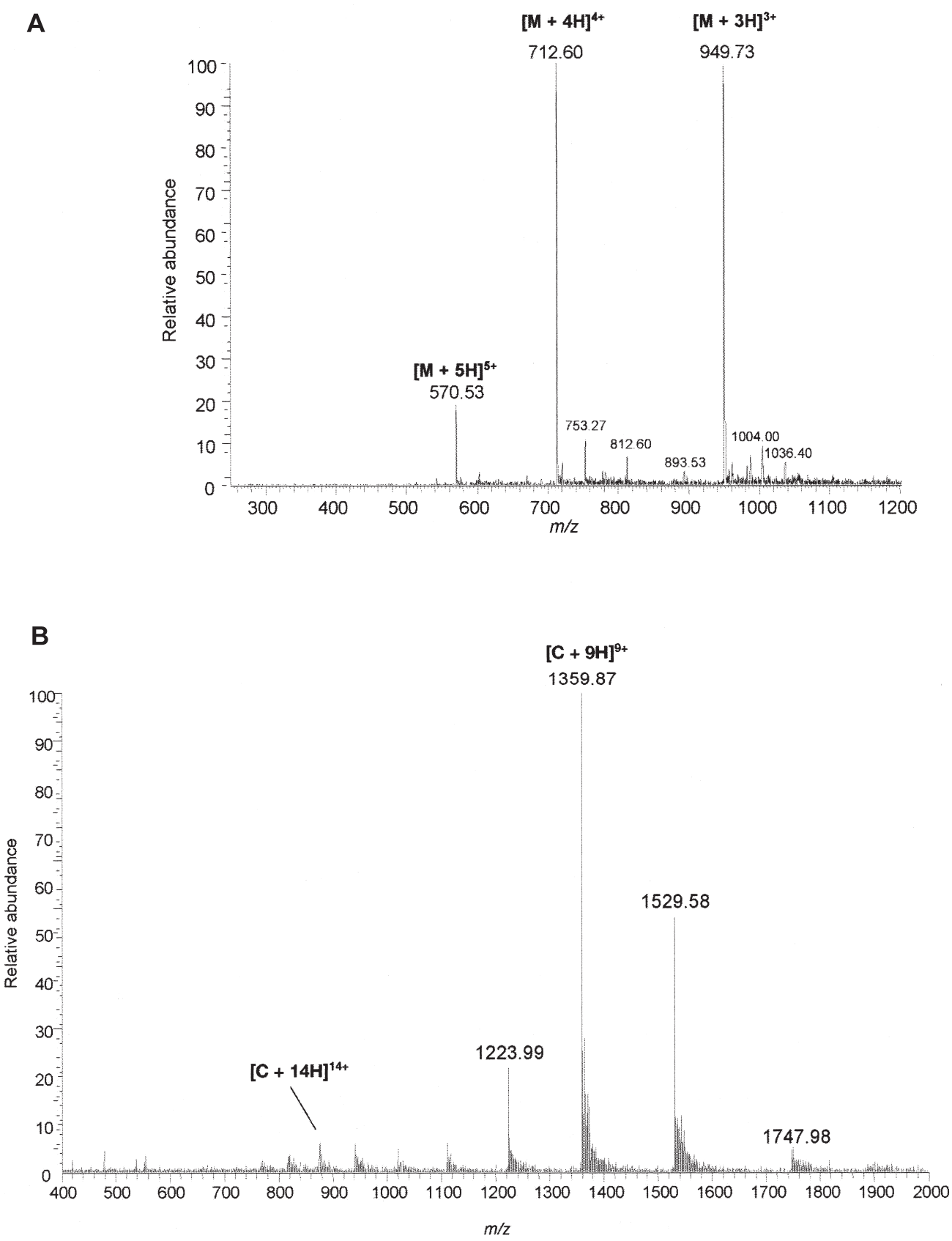

FIGURE 5. Examples of DESI mass spectra. (A) DESI mass spectrum of 100 pg (total amount deposited on surface) of melittin (M) (molecular mass 2847 Da) deposited on a polyvinylidene difluoride (PVDF) surface. (B) DESI mass spectrum of 1 pmol of cyctochrome $c(C)$ (from equine heart) on a PTFE surface. Methanol: $\mathrm{H}_{2} \mathrm{O}(1: 1)$ was used as the spray solvent at a flow rate of $5 \mu \mathrm{L} / \mathrm{min}$. ( $\mathrm{m} / \mathrm{z}$ ) Mass-to-charge ratio. For more details, see Desorption Electrospray Ionization (DESI) Analysis of Intact Proteins/Oligopeptides. (Reprinted with permission, (c) 2007 Scion Publishing Ltd.)

\section{APPLICATIONS AND METHODS}

Application areas of DESI can be divided into two categories, based on the type of sample preparation method used. The first category covers the investigation of pre-deposited samples; for example, where solutions are deposited on substrates like paper or plastic. One of the important features of this type of DESI experiment is its high-throughput character, which has been used in the analysis of biological samples as well as pharmaceutical formulations (Chen et al. 2005; Weston et al. 2005). This type of sample and the high-throughput character of the application have potential value in the fields 
of industrial process monitoring, food safety, environmental analysis, and clinical diagnostics. Highthroughput DESI analysis of biological fluid samples (e.g., urine) for metabolite identification is also receiving attention because of the potential value of information on the distribution of characteristic small molecules as biomarkers of disease. With limited sample preparation required even for the analysis of raw urine, a few hundred samples per hour can be analyzed using DESI-mass spectrometry (DESI-MS) when biological fluids are spotted onto paper or other suitable substrates. In work by Chen et al. (2005), the detection of more than 80 metabolites in urine without any sample preparation was achieved using DESI.

The second group of applications deals with the DESI-MS investigation of natural, unmodified surfaces. One of the most important applications of this type is the investigation of biological tissues. Thin tissue sections prepared for microscopy or native, freshly cut tissue surfaces are used as such for DESIMS analysis and even-using a fine DESI probe beam-chemical imaging. Imaging MS based on MALDI and SIMS has become a powerful technique for analyzing histological sections of biological tissues (Caprioli et al. 1997; Pacholski and Winograd 1999). These techniques require that the sample be confined to the high-vacuum region of the instrument, severely limiting any further chemical or physical manipulation of the sample, a limitation not inherent in DESI studies. The determination of the spatial distribution of natural tissue components such as membrane phospholipids has recently been demonstrated using DESI (Wiseman et al. 2005), and in other work an automated DESI source was used for two-dimensional (2D) imaging of tissue sections and other surfaces (Wiseman et al. 2006; Ifa et al. 2007). Other applications of DESI to natural surfaces occur in forensic analytics, especially the detection of explosives, toxins, and drugs of abuse on the surfaces of personal items.

\section{REFERENCES}

Caprioli, R.M., Farmer, T.B., and Gile, J. 1997. Molecular imaging of biological samples: Localization of peptides and proteins using MALDI-TOF MS. Anal. Chem. 69: 4751-4760.

Chen, H., Talaty, N.N., Takats, Z., and Cooks, R.G. 2005. Desorption electrospray ionization mass spectrometry for high throughput analysis of pharmaceutical samples in the ambient environment. Anal. Chem. 77: 6915-6927.

Ifa, D.R., Wiseman, J.M., Song, Q., and Cooks, R.G. 2007. Development of capabilities for imaging mass spectrometry under ambient conditions with desorption electrospray ionization (DESI). Int. J. Mass Spectrom. 259: 8-15.

Pacholski, M.L. and Winograd, N. 1999. Imaging with mass spectrometry. Chem. Rev. 99: 2977-3006.

Venter, A., Sojka, P.E., and Cooks, R.G. 2006. Droplet dynamics and ionization mechanisms in desorption electrospray ionization mass spectrometry. Anal. Chem. 78: 8549-8555.
Vincenti, M. and Cooks, R.G. 1988. Desorption due to charge exchange in low-energy collisions of organofluorine ions at solid surfaces. Org. Mass Spectrom. 23: 317-326.

Weston, D.J., Bateman, R., Wilson, I.D., Wood, T.R., and Creaser, C.S. 2005. Direct analysis of pharmaceutical drug formulations using ion mobility spectrometry/quadrupole-time-of-flight mass spectrometry combined with desorption electrospray ionization. Anal. Chem. 77: 7572-7580.

Wiseman, J.M., Puolitaival, S.M., Takats, Z., Cooks, R.G., and Caprioli, R.M. 2005. Mass spectrometric profiling of intact biological tissue by using desorption electrospray ionization. Angew. Chem. Int. Ed. Engl. 44: 7094-7097.

Wiseman, J.M., Ifa, D.R., Song, Q., and Cooks, R.G. 2006. Tissue imaging at atmospheric pressure using desorption electrospray ionization (DESI) mass spectrometry. Angew. Chem. Int. Ed. Engl. 45: 7188-7192. 


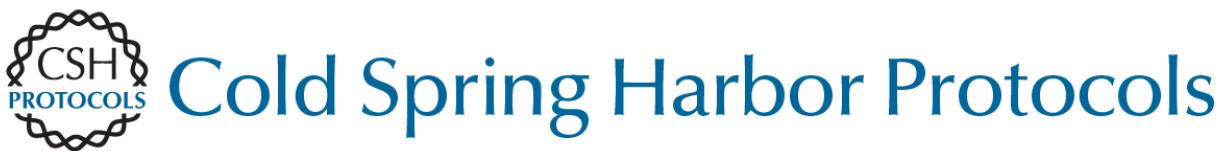

\section{Desorption Electrospray lonization: Proteomics Studies by a Method That Bridges ESI and MALDI}

\author{
Zoltan Takats, Justin M. Wiseman, Demian R. Ifa and R. Graham Cooks
}

Cold Spring Harb Protoc; doi: 10.1101/pdb.top37

\begin{tabular}{|c|c|}
\hline $\begin{array}{r}\text { Email Alerting } \\
\text { Service }\end{array}$ & Receive free email alerts when new articles cite this article - click here. \\
\hline $\begin{array}{l}\text { Subject } \\
\text { Categories }\end{array}$ & $\begin{array}{l}\text { Browse articles on similar topics from Cold Spring Harbor Protocols. } \\
\text { Characterization of Protein Complexes ( } 83 \text { articles) } \\
\text { Characterization of Proteins (208 articles) } \\
\text { Chromatography (47 articles) } \\
\text { Chromatography, general (134 articles) } \\
\text { High-Throughput Analysis, general (155 articles) } \\
\text { High-Throughput Chromatography (4 articles) } \\
\text { Liquid Chromatography (51 articles) } \\
\text { Mass Spectrometry (78 articles) } \\
\text { Protein Identification and Analysis (202 articles) } \\
\text { Proteins and Proteomics, general (575 articles) } \\
\text { Proteome Analysis (56 articles) } \\
\text { Proteomics (66 articles) } \\
\text { Structural Analysis ( } 16 \text { articles) }\end{array}$ \\
\hline
\end{tabular}

